\title{
CONGENITAL ANOMALIES AND ABNORMALITIES OF THE URINARY TRACT.
}

\author{
By OSWALD L. ADDISON, F.R.C.S. \\ (Emeritus Surgeon, West London Hospital; Consulting Surgeon, Hospital for Sick Children, \\ Great Ormond Street.)
}

\section{INTRODUCTION.}

Congenital abnormalities and malformations are more frequent in the urinary tract than in any other system of the body, and it is an interesting and important fact that they are very commonly multiple. It is quite usual to find both kidneys and ureters involved, or to find bladder and penile deformities with faulty development of one or both kidneys and ureters. Many of them cause little or no disability and are discovered incidentally during a urological or post-mortem examination. On the other hand, some few are incompatible with life, and by far the greater number gives rise to serious disabilities, chronic illnesses and often a shortened life.

In an article of this length it is impossible to give an account of all the various abnormalities that may occur and have been recorded from time to time, nor is it necessary for our purpose, and I propose therefore to consider only the more common abnormalities; those which are the causes of serious disabilities or illnesses, or give rise to difficulties and dangers in the diagnosis and treatment of urinary disorders.

The possible methods of development of the various abnormalities though of great interest are of no practical value from the point of view of treatment and will not be considered. Most of these conditions give rise to troubles in early life, even in infancy, only a small proportion of these cases reaching adult life before symptoms arise. The amount of illness in childhood directly due to malformations of the urinary tract is very large indeed. Practically all the chronic infections of the urinary tract, with the exception of the rare cases of tubercle, and those associated with calculi, are directly due to congenital malformations. Although these abnormalities have been known for years, their importance as the prime factor in chronic infection has not been appreciated. This failure to recognize their importance has been due almost entirely to the fact that until recently it has been impossible to investigate these cases on proper urological lines. Now, with modern instruments, and radio-opaque drugs both for intravenous and ureteric pyelography that can be used safely in children, it is quite possible to make a complete urological examination even in an infant. As a result knowledge of these cases is rapidly growing and with increasing experience, further important developments in treatment may be expected.

\section{CONGENITAL ABNORMALITIES AND MALFORMATIONS OF THE KIDNEYS.}

Abnormalities and malformations of the kidneys are extremely common and of great variety. Their frequency has been estimated at $3-4 \%$ of all cases examined post mortem. 
Anomalies of Number and Position. A third kidney has occasionally been recorded. Absence of both kidneys is most frequently found in anencephalic and other types of monster, and is of no importance. Cases of solitary kidney are of sufficient frequency to make recognition of the fact of the greatest importance. Ballowitz found I2 cases in 28,423 autopsies. A solitary kidney is always hypertrophied and is not incompatible with an ordinary life, and indeed has most often been discovered accidentally at autopsy. A solitary kidney is said to be more liable to disease than a normal one; 79 out of I70 were found by Anders to be diseased. There seems to be a special liability to the formation of calculus, and cases of tuberculosis, hydronephrosis, and malignant disease have been recorded. It is frequently associated with malformations of the genital system.

The chief importance of a single kidney lies in its recognition before any renal operation is undertaken. There have been numerous cases of nephrectomy of a solitary kidney. Fortunately the absence of a second kidney is nowadays readily discovered by cystoscopy and pyelography. On cystoscopy, a normal ureteric orifice may be present on the side of the absent kidney and the ureter may be patent for a short distance, but no contractions of the orifice occur. The ureteric orifice may be absent, or represented by a dimple, and occasionally the corresponding half of the trigone may be absent.

In any case of doubt as to the presence of two kidneys an intravenous pyelogram should be done, when the presence or absence of any secretion on the suspected side will settle the question.

Horseshoe Kidney. Fusion of the kidneys has been recorded in a great variety of forms, the commonest being the horseshoe type. The frequency of this type of fused kidney has been estimated at $I-I, 000$ by Morris. The two kidneys are joined together by their lower poles across the front of the aorta and vena cava; rarely cases have been recorded where the junction was between the upper poles. The union is of varying thickness and may be of fibrous tissue only, but usually consists of renal parenchyma, and may be large enough to be felt lying across the front of the vertebræ.

The horseshoe kidney varies much in size and is situated rather lower than the normal kidneys, and the two halves are rather nearer the middle line. The ureters nearly always pass over the front of the isthmus, but they have been recorded passing behind, and a third ureter coming from the isthmus has been found.

A horseshoe kidney may give rise to no symptoms but there is a great liability to the development of hydronephrosis of one or both halves, from compression of the ureter as it crosses over the isthmus, though the hydronephrosis may be associated with a megalo-ureter, in which case both are probably caused by a defect in the neuromuscular development of the pelvis and ureter.

A horseshoe kidney is readily recognized on pyelography by the fact of the lower calices on either side being nearer the middle line than normal and pointing inwards; the calices of the upper poles may also point a little towards the middle line. The two pelves do not communicate across the middle line. Occasionally one may find a normally situated kidney with the pelvis lying in front and the calices of the upper and lower poles pointing inwards. 
Heminephrectomy for growth, calculus, tubercle, etc., is not usually difficult and may be safely undertaken, provided that the function of the other half of the kidney is satisfactory.

Disc Kidney. The kidneys are completely fused, and form a lobulated mass, usually low down in the middle line about the level of the bifurcation of the aorta. There are usually two ureters.

Unilateral fused Kidneys. In this condition the double kidney lies to one side of the vertebral column, the upper pole of one kidney being joined to the lower pole of the other. The pelves may both look towards the middle line, or may face in opposite directions. There is no regular arrangement of the ureters, which may run parallel, or cross each other to open on opposite sides of the bladder. Both ureters have been found entering the same side of the bladder.

Misplaced or Ectopic Kidneys. Misplacement of one kidney is more frequent than misplacement of both, and usually the kidney is placed lower down on its own side, in the iliac fossa, or in the pelvis. The ectopic kidney is nearly always malformed and like the horseshoe kidney is likely to become infected or hydronephrotic. When lying in the pelvis in women it is said to be liable to cause disorders of menstruation, and increased frequency of micturition, and if hydronephrotic, may interfere with parturition.

An ectopic kidney is readily recognized by pyelography.

A pelvic kidney may be removed through a midline incision, either transperitoneally, or extraperitoneally by displacing the peritoneum laterally. If the kidney is much inflamed and adherent, the operation may be very difficult owing to the irregularity of the blood vessels supplying it, and to the proximity of the iliac and other vessels.

Congenital Cystic Kidney. It is now generally accepted that this condition is the same as the polycystic kidney of adults. The kidney consists of a series of cysts varying in size from a pin's head to a grape. The cysts are yellowish, or brown and translucent, and filled with a watery fluid containing epithelial cells, traces of urea and chlorides, albumin, casts, etc. On sections, little or no kidney substance can be seen, but the cysts are separated by fine fibrous septa in which may be found tubules and compressed glomeruli. The pelvis and calices are greatly attenuated and dragged out, and form a fine branching system which gives a characteristic pyelogram quite unlike that seen in any other renal condition. (Fig. I). Owing to the extremely poor concentration of the urine an intravenous pyelogram is not very satisfactory, and a ureteric pyelogram is usually necessary.

The condition was found I6 times in I0,177 autopsies by Naumann, and is nearly always bilateral though generally much more advanced on one side than the other. Lejars found only two out of 63 cases were unilateral. Though comparatively few cases are seen in childhood, it may be present at birth and the enlargement of the kidney so great as to obstruct labour.

Symptoms. In childhood the symptoms are not marked, and the condition is usually discovered on the routine examination of the abdomen in a child who is not doing well. In adults, the symptoms which usually begin between the ages 
of 40 and 60 are more definite; a dull aching pain is frequent, while attacks of renal colic with hæmaturia are common. Polyuria of low specific gravity, and generally a trace of albumin, with diminution of urinary salts is usual.

On examination one or both kidneys are felt to be enlarged, as a rule, one much larger than the other, even filling the greater part of the abdomen. In a thin patient the lobulations on the surface of the kidney may be felt and where, as is generally the case, both kidneys are enlarged the diagnosis is not difficult, but if the enlargement is unilateral, it is liable to be mistaken for a growth, or hydronephrosis. A ureteric pyelogram, however, will make the diagnosis clear. The disease is ultimately fatal, the patient dying of uræmia, but the condition may last for many years, and cases over 70 and 80 years of age have been recorded.

No treatment is of value, and nephrectomy except in the very rare instance of unilateral disease can only do harm, by diminishing the already inadequate amount of active renal tissue.

\section{ABNORMALITIES OF THE RENAL VESSELS.}

Irregularities of the arteries and veins are frequent; the aberrant branches rising from the aorta, common, internal iliac, and inferior mesenteric arteries, such irregularities being the rule in the case of horseshoe and ectopic kidneys. Irregular arrangement of vessels with a normal kidney is of importance in two ways. An artery of considerable size may enter the upper pole of the kidney, and if not recognized may cause serious difficulty during an operation. Similarly, an artery may enter the lower pole, and is frequently associated with a hydronephrosis, the vessel apparently constricting the pelvi-ureteric junction. It is important to remember that ligature of an aberrant vessel, if of any size, may be followed by a large area of necrosis and deaths from this cause have been recorded.

\section{ABNORMALITIES OF THE PELVIS AND URETER.}

Variations and irregularities of the normal development of the pelvis and ureter are undoubtedly the most common of all urinary congenital abnormalities and while many of them are found accidentally and cause no symptoms, they are more frequently the cause of urinary disease than any other abnormality. A double pelvis is not uncommon. Absence of one or both ureters has been recorded, generally with absence of the corresponding kidney or kidneys. Occasionally a hydronephrosis present at birth, has been found with absence of the corresponding ureter, or the ureter represented by a fine fibrous cord.

Hydronephrosis and Dilatation of the Ureters. Hydronephrosis which may be unilateral, or bilateral, is caused by some obstruction to the urinary outflow, though in some of the bilateral cases no mechanical obstruction can be found. Hydronephrosis may be " closed " "intermittent" or " open."

A. Closed hydronephrosis is usually caused by an atresia at the ureteropelvic junction. (Fig. 2). The closure may be complete at birth, or if partial, become complete later from kinking of the ureter caused by the drag of the enlarging hydronephrosis. The ureter may be completely absent or represented by a fibrous cord. Ureteric valves at the ureteropelvic junction may also cause hydronephrosis. This type of hydronephrosis is generally unilateral, and may be of large size, 
often filling more than half the abdomen; it may be large enough to cause difficulty at birth.

B. Intermittent. This form may be unilateral or bilateral, and seldom reaches a very large size. The obstruction causing it may ultimately become complete, and lead to a "closed" hydronephrosis. Congenital atresia, valves at the ureteropelvic junction, and aberrant vessels looping up the pelvis are the usual causes. (Fig. 3). Strictures lower down in the ureter may at times cause the condition, and in this case there is an associated dilatation of the ureter. The most typical example of this condition is seen when it is caused by an aberrant renal vessel running to the lower pole of the kidney, and crossing usually in front of the ureteropelvic junction. The ureter becomes kinked and often bound to the pelvis by adhesions.

Any sudden increase in urinary secretion may cause a temporary block with severe renal colic, which unlike the sudden colic due to calculus begins gradually. The kidney can often be felt to be enlarged and tender during the attack.

C. "Open." This type is nearly always bilateral and seldom reaches a large size though the ureters are often relatively enormous. It is generally caused by some obstruction of the bladder outlet, or by urethral valves in the prostatic urethra. In some cases no obstruction has been found and the condition has been called idiopathic. (Fig. 4).

Horseshoe Kidney and Hydronephrosis. Hydronephrosis caused by compression of the ureter over the isthmus of a horseshoe kidney is frequently found, and may reach a very large size. It is more or less centrally situated and may be mistaken for a distended bladder.

The symptoms caused by a hydronephrosis are:-

I. Discomfort or pain due to distension of the sac.

2. Renal colic (in the intermittent type).

3. Renal insufficiency and uræmia.

4. Infection and pyuria.

Infection, often of a severe grade, sooner or later occurs in nearly all cases, though with the "closed" and "intermittent" types it may not occur before adult life. In the "open" type infection occurs early, often during the first few months of life and is of a severe grade.

Supernumerary Ureters. A double ureter may be present on one or both sides, and may be complete or incomplete. Junction of the two ureters may take place anywhere between the kidney and bladder. Though it is more common for them to join than to open separately into the bladder; two ureteric openings on one side of the bladder are not very rare. On the other hand, four entirely separate ureters with four separate openings into the bladder is extremely rare.

In every case of double ureter, each ureter comes from its own pelvis and the pelves do not communicate with one another, an important point to remember, for it is quite possible to have a pyonephrosis of one pelvis while the other remains healthy, and a heminephrectomy may be quite feasible. 
Double pelves may always be recognized in a pyelogram even when only one is satisfactorily shown, the pelvis is elongated and the calices deformed and rather clubbed.

Aberrant Implantation of Ureter. Extravesical ending of the ureter has been recorded on a number of occasions both in the male and female, the abnormal ureter not infrequently being an extra one. In the male the ureter has most often been found opening into the prostatic urethra, and has been recorded opening into the vas deferens, seminal vesicle, or ejaculatory duct. In the female the aberrant opening has been found most often in the vagina, but may occur in the vestibule, or urethra, and has been recorded in the uterus, and fallopian tubes.

In the male the condition does not give rise to symptoms of incontinence as the opening is above the external sphincter, but in the female when the opening is in the vagina, or vestibule, incontinence is the rule and it is usually present when the opening is in the urethra. In the female constant dribbling of urine, with at the same time perfect bladder control and normal power of micturition, is practically certain evidence of extra vesical implantation.

Obstructions and Dilatations of the Ureter.

Congenital strictures of the ureter, already referred to as a frequent cause of hydronephrosis, occur in any part of its course; they vary considerably in length and may be multiple. The muscles are replaced by fibrous tissues. Strictures are most often found at the pelvi-ureteric junction or in the bladder wall. Ureteric valves are another cause of obstruction and are found at the ureteropelvic junction. They are due to the persistence of folds of the mucous membrane, which are normally present in the foetus, and disappear after three months. Kinks of the ureter at the ureteropelvic junction causing obstruction have been recorded in the case of ectopic kidneys, but with a normally situated kidney a kink when present is secondary to some local inflammatory condition causing adhesions, or to the looping up of the pelvis by aberrant vessels. Sharp angulations and S-shaped bends are commonly present in elongated and dilated ureters, but do not cause obstruction.

Stenosis of the ureteric opening in the bladder, causing a prolapse of the mucous membrane and the formation of a ureterocele, is another cause of ureteric obstruction. The ureterocele which consists of two layers of prolapsed mucous membrane, ureteric and bladder, forms a smooth, glistening, semitranslucent swelling, over the site of the ureteric orifice, and is usually the size of a grape but may be considerably larger. (Fig. 5). In the female it sometimes prolapses down the urethra, and shows as a small cyst between the labia.

The appearance as seen on cystoscopy is quite characteristic, and the pinhole opening of the ureter may sometimes be seen on the surface of the cyst. At times the cyst can be seen to fill and empty as the peristaltic waves pass down the ureter. Ureterocele is not infrequently associated with a double ureter, the opening of one ureter being stenosed, and the opening of the second being hidden by the ureterocele.

Congenital strictures may cause atrophy of the kidney, but more frequently cause hydronephrosis, and dilatation of the ureter above the stricture. Many cases of congenital dilatation of the ureter are seen where no obstruction can be found, they may be unilateral or bilateral and a hydronephrosis of the corres- 
ponding kidney may or may not be present, but there is usually some pelvic dilatation. (Fig. 4). Presumably this type of megalo-ureter is due to failure of the neuromuscular development.

Of all the abnormalities and deformities of the urinary tract, there is no doubt that strictures and dilatations of the ureters are the most important, from their very great frequency and the fact that sooner or later they cause damage to the kidney. Meredith Campbell found 42 cases of gross ureteral blocking in 2,420 autopsies on children at the Bellevue Hospital; an incidence of nearly two per cent. In addition to the back pressure effects on the kidney, in consequence of the stagnation of urine, infection often of a severe grade is only a question of time. By far the greater number of these cases gives trouble in early childhood or infancy, and few reach adult life before symptoms arise. These cases of dilated pelves and ureters are very liable to be complicated by the presence of calculi, and it is much more common in children to find a calculus in a congenitally dilated ureter, than it is to have a dilatation of the ureter secondary to a calculus.

Chronic pyuria with frequent exacerbations is extremely common in early childhood, and it is no exaggeration to say that ninety-nine out of every hundred cases are due to some delay in emptying of the ureter, either from a congenital stricture, or dilatation with an imperfect musculature. It is not generally recognized even, yet that every case of intermittent or chronic pyuria in childhood must have a complete urological investigation, and that there is no such condition as "chronic pyelitis". It is extremely doubtful if there is such a lesion as "acute pyelitis"; the lesion is almost certainly a general infection of the kidney.

Symptoms in these cases vary from a mild persistent bacilluria, and pyuria, with general ill-health and irregular febrile attacks, to a constant heavy pyuria with attacks of fever and severe pain; the deposit of pus aften diminishing at the onset of the attack, and a large increase in quantity coinciding with the subsidence of the pain and temperature.

The treatment of these cases necessarily depends on the nature of the lesion, whether there is more than one and whether both sides are involved.

Ureteroceles and ureteral strictures in the bladder wall can be readily dealt with by operation, but plastic operations on the kidney pelvis and upper end of the ureter are seldom satisfactory, especially in children when most of these cases come under treatment. Dilatation of strictures by ureteric bougies can seldom be accomplished in children. In cases of gross infection and dilatation of the kidney and ureter, nephrectomy when practicable is the best treatment. In some of the milder degrees of infection pelvic lavage may be tried, but this is a difficult operation in the child and any benefit that is gained is purely temporary. Many of the milder cases can be helped by hexamine, and sometimes by a course of vaccines, but a ketogenic diet is of no value in the infections of the kidney and ureters consequent upon congenital malformations.

\section{MALFORMATIONS OF THE BLADDER.}

Urachus. The urachus is the remains of the allantoic stalk and extends from the apex of the bladder to the umbilicus. Occasionally it remains patent for its whole length, and there is a constant escape of urine from the umbilicus; 
at other times it is closed at the ends and a cyst, sometimes of large size, develops between the peritoneum and the anterior abdominal wall.

Two types of double bladder have been recorded, in one form the division has been into two lateral halves, and in the other into anterior and posterior. The division may be incomplete, and a double bladder with two urethræ has been found.

Diverticula of congenital origin are not infrequent and are usually found opening onto the base or lateral walls. They may reach a large size and though they seldom give trouble in childhood, in adult life complications are frequent. Most often the symptoms are those of cystitis with residual urine containing a large amount of pus; calculi frequently form in the sac, and where the diverticulum is situated near the ureteric orifice, the ureter may be obstructed with resulting back pressure effects on the ureter and kidney.

On cystoscopy, diverticula are readily recognized as round, dark, openings in the bladder wall, and an estimate of their size can be obtained from a cystogram.

Obstruction at the Internal Meatus.

A submucous fibrosis of the bladder outlet has been recorded by Meredith Campbell in male infants, symptoms of difficult micturition and retention, often being present from birth, or occurring during the first few years; in others, symptoms may be delayed till adult life. In twenty out of three hundred and fifty-five cases the bladder-neck fibrosis recorded by Young the lesion was considered to be congenital.

Ectopia Vesicae. This is a condition in which the anterior vesical wall and part of the abdominal wall are absent. The posterior wall of the bladder and trigone are exposed on the surface. It is found in both sexes but is more common in males, and appears above the pubis as a raised red area of mucous membrane on which the openings of the ureters can be seen. It is associated with a malformed epispadac penis and absence of the symphysis. Nondescent of the testes is a usual accompaniment, and the ureters and kidneys are frequently malformed. Ascending infection of the ureters occurs sooner or later, and Buerger records that only 23 of 74 untreated cases reached 20 years of age.

Many forms of plastic operation have been devised to deal with the defect by turning in skin flaps to cover the raw surface, and form some sort of receptacle for the urine, but none of them has proved satisfactory. The flaps constantly break down, and operations have to be repeated many times to get even a partial success, and then the patient still has no control. Later the formation of phosphates on the skin flaps is a constant source of trouble and distress.

The most satisfactory method of treatment is by transplantation of the ureters into the rectum, or sigmoid. Of the various ways of doing this, the operations of Maydl and Sir Harold Stiles are the most satisfactory. In Maydl's method an oval area of bladder wall containing the ureters is freed from the rest of the bladder and transplanted into the sigmoid. In the operation devised by Stiles, the ureters are dissected out, and transplanted separately into the lowest part of the colon. The second ureter is transplanted some weeks after the first and the operation should be done about the age of six or seven. Infection of the kidneys follows almost certainly in every case and ingenious methods for making 
valvular openings into the bowel with the hope of preventing this have been devised, notably by Coffey. Although the danger of pyelonephritis is a very real one, many of these patients establish a tolerance to the infection and grow up to lead healthy and useful lives. Rectal control is soon established and urine can generally be retained for three or four hours. Grey Turner published a very valuable account of the operation with a list of cases in the "British Journal of Surgery," I929.

\section{PENIS AND URETHRA.}

Epispadias. The urethra opens on the dorsal surface of the penis; three degrees are recognized, first a cleft only in the glans, second a cleft extending the whole length of the penis, third a cleft of the whole length associated with ectopia vesicæ. In the second and third degrees there is no sphincteric control. Plastic operations to form a sphincter have not proved successful, and the question of ureteric transplantation should be considered.

Hypospadias. According to their position ventral clefts of the urethra are divided into (I) glandular, (2) penile, and (3) perineal.

In glandular hypospadias the urethra ends at the base of the glans, which is generally grooved. In the perineal type the opening is between the scrotum and anus, the scrotum is in two halves and the testes generally undescended. As the penis is generally very ill-developed and tied down, this type is very liable to be mistaken for hermaphroditism.

The glandular type of hyposadias seldom requires operation, disability of micturition is slight and fertility is unaffected. In the penile, penoscrotal and perineal forms numerous operations have been devised, and all, with two exceptions, give very unsatisfactory results. Bucknall's operation for hypospadias at the penoscrotal junction is useful, but has the disadvantage that the skin of the scrotum, which is used to form the floor of the urethra, is liable to grow hairs at puberty. This inevitably leads to phosphatic calculi being deposited on them. Perhaps the growth of hair might be prevented by X-ray treatment.

For all types the operation of Arthur Edmunds, is infinitely to be preferred The operation is done in three stages, and the results are excellent.

Urethra. A few cases of complete duplication of penis and urethra have been described. Accessory urethræ in great variety have been recorded, usually opening somewhere about the base of the glans, but an opening at the root of the penis has been found.

They are found either above or below the true urethra, the dorsal form being separated from it by the corpora cavernosa. The dorsal form generally ends blindly about the symphysis, but may enter the bladder or divide and enter the ejaculatory ducts. The ventral form is less common usually commencing about the bulb and opening on the glans, but it may have an opening at the penoscrotal angle.

Accessory urethræ as a rule cause no trouble except in cases of urethral infection. 
Pin-hole Meatus. Stenosis of the meatus is a common and troublesome condition, which causes difficulty in micturition, and not infrequently retention. Meredith Campbell records a case where dilatation of the urethra occurred with dilatation of the bladder and back pressure effects on the kidneys. Treatment consists in dividing the opening downwards, but the enlarged meatus must be kept dilated by passing a bougie daily until healed, otherwise the opening will rapidly close.

Urethral Valves and Diaphragms. Urethral valves are by far the most important of the congenital abnormalities of the urethra on account of the very serious results produced by them.

The condition was first described by Langenbeck in I802 as a post-mortem finding, but it was not until I9I3 that it was first diagnosed clinically, and treated by Prof. H. Young of the Johns Hopkins Hospital. Since that time many cases have been recorded by Young, Meredith Campbell, and others.

The valves are formed of mucous membrane and are found in the posterior urethra. Professor Young describes three types, the first two in connection with the verumontamum, while the third forms a partial diaphragm with a central opening, and may be found at different levels in the posterior urethra.

The first two types spring from the upper or lower ends of the verumontamum and form folds extending round the sides of the urethra to the anterior surface. The folds may be single, or double, and represent exactly the valves of the veins, (Figs. 6 and 7). Unless double and the opening between them very small, they offer no obstruction to the passage of a catheter. The diaphragm type can be recognized as a definite obstruction on passing a bougie. The first two forms are necessarily found only in males, but the diaphragm type has also occasionally been recorded in girls. (Fig. 8).

Generally these cases die of uræmia in infancy, or during the first few years, but if the obstruction is not of a severe grade they may go on to adolescence, or adult life, though if untreated all ultimately die from the effects of back pressure on and infection of the kidneys.

The clinical picture of these cases is characteristic. A male child or infant is brcught up with a history of never having been able to pass urine properly since birth. The stream has always been weak or a mere dribble, later continuous enuresis develops. On examination, the child is found to be small and undeveloped for his age, pale and listless, and often very thirsty and showing signs of uræmia. The clothes are soaked with urine, the bladder distended to the umbilicus or higher, and urine is dripping from the penis. The urine is of low specific gravity, contains a cloud of albumin and pus, and is nearly always heavily infected with B.coli even in the first few months of life. The blood urea and renal function tests show that the kidneys are badly damaged.

Treatment consists in gradual decompression of the distended bladder followed by destruction of the valve. The valve may be destroyed by a special punch, invented for the purpose by Young, or by the retrograde passage of bougies through a suprapubic opening. Young, Campbell and others have reported a number of successful cases, but the majority of those seen in this country are moribund when first brought to hospital. 